\title{
BMJ
}

\section{Self monitoring of blood glucose in type 2 diabetes: longitudinal qualitative study of patients' perspectives}

\author{
Elizabeth Peel, lecturer in psychology, ${ }^{1}$ Margaret Douglas, consultant in public health medicine, ${ }^{2}$ \\ Julia Lawton senior research fellow ${ }^{3}$
}

${ }^{1}$ Psychology, School of Life and Health Sciences, Aston University, Birmingham B4 7ET

${ }^{2}$ Lothian NHS Board,

Deaconess House, 148 Pleasance, Edinburgh EH8 9RS

${ }^{3}$ Research Unit in Health, Behaviour and Change,

Clinical Sciences and Community Health, Medical School,

University of Edinburgh, Edinburgh EH8 9AG

Correspondence to: $E$ Peel

e.a.peel@aston.ac.uk

doi:10.1136/bmj.39302.444572.DE

\section{ABSTRACT}

Objective To explore views of patients with type 2 diabetes about self monitoring of blood glucose over time.

Design Longitudinal, qualitative study.

Setting Primary and secondary care settings across Lothian, Scotland.

Participants 18 patients with type 2 diabetes.

Main outcome measures Results from repeat in-depth interviews with patients over four years after clinical diagnosis.

Results Analysis revealed three main themes-the role of health professionals, interpreting readings and managing high values, and the ongoing role of blood glucose self monitoring. Self monitoring decreased over time, and health professionals' behaviour seemed crucial in this: participants interpreted doctors' focus on levels of haemoglobin $A_{1 c}$, and lack of perceived interest in meter readings, as indicating that self monitoring was not worth continuing. Some participants saw readings as a proxy measure of good and bad behaviour-with women especially, chastising themselves when readings were high. Some participants continued to find readings difficult to interpret, with uncertainty about how to respond to high readings. Reassurance and habit were key reasons for continuing. There was little indication that participants were using self monitoring to effect and maintain behaviour change.

Conclusions Clinical uncertainty about the efficacy and role of blood glucose self monitoring in patients with type 2 diabetes is mirrored in patients' own accounts. Patients tended not to act on their self monitoring results, in part because of a lack of education about the appropriate response to readings. Health professionals should be explicit about whether and when such patients should self monitor and how they should interpret and act upon the results, especially high readings.

\section{INTRODUCTION}

Effective self management is considered the cornerstone of successful diabetic control, and self monitoring of blood glucose may have a role in this. For type 2 diabetes, however, there is still no firm agreement among diabetes health professionals ${ }^{1-3}$ and in the research literature ${ }^{4-9}$ about the role and value of self monitoring.
Despite the lack of conclusive evidence of an association between self monitoring of blood glucose and glycaemic control (even in large scale observational studies with heterogeneous groups of patients ${ }^{10-12}$ ) and findings that self monitoring may lead to anxiety, ${ }^{13}$ clinical practice guidelines often promote self monitoring by patients with type 2 diabetes. They stress that it "can be useful in preventing hypoglycaemia and adjusting medications, medical nutritional therapy, and physical activity." 14 They often refer to research that supports self monitoring: for example, the Canadian Diabetes Association ${ }^{15}$ cites one of the few observational studies ${ }^{16}$ that showed it was associated with lower concentrations of haemoglobin $\mathrm{A}_{1 \mathrm{c}}$ in diabetic patients not treated with insulin. The UK National Institute for Clinical Excellence (NICE) contended that "self monitoring should be taught if the need/purpose is clear and agreed with the patient. Self monitoring can be used in conjunction with appropriate therapy as part of integrated selfcare." 17 Thus the recommendation to self monitor appears in guidance to doctors, although its clinical benefit remains inconclusive. ${ }^{18}$

We assessed the views of patients with newly diagnosed diabetes about self monitoring of blood glucose, since they are responsible for taking and responding to readings on a daily basis, and found that they held positive and negative views. ${ }^{19}$ Positive aspects were that, in the absence of symptoms or complications, self monitoring provided patients with evidence that they had diabetes, and low readings offered reassurance and promoted a sense of "success." Although self monitoring could increase awareness of the impact of diet on blood glucose levels, it also created a sense of failure if readings remained high. High readings that were counterintuitive posed particular problems for patients and could lead to abandonment of dietary regimens. These findings suggested that patients with well controlled diabetes viewed self monitoring positively, but patients with poorly controlled diabetes were likely to voice concerns. Our research also identified early signs of "monitoring fatigue" in these patients.

As our study was restricted to patients with newly diagnosed diabetes, we undertook a follow-up study to gain a longitudinal perspective of the patients' 
views and use of blood glucose self monitoring. Our aims were to explore patients' experiences and views of self monitoring over time, responses to high or low readings, reasons for increasing or decreasing self monitoring, and views of the advice and feedback received from health professionals. This analysis forms part of a broader study examining the views of patients with type 2 diabetes about health service delivery and their experiences of managing their disease. ${ }^{20-25}$

\section{METHOD}

In 2002-3, we recruited 40 patients who had received a clinical diagnosis of type 2 diabetes within the previous six months and interviewed them three times over the following year. The sample was selected to reflect the demographic spread of people with type 2 diabetes in Lothian and Scotland and to include patients receiving diabetes care in hospital and in general practice. The

Box 1 The role of health professionals on participants' views of blood glucose self monitoring
Participant F8.4:
Q: So did they ever ask to see your readings that you'd taken?
A: Oh no, no. I don't even think they've asked me if I've got a meter.
Q: So they've not shown much interest in that really?

A: Well I suppose they only go on their own.

Q: So, like we talked a bit about how you'd use the blood glucose meter and what are you doing at the minute in terms of that, have you stopped doing it completely?

A: Yes, stopped totally because, whereas I thought I was doing quite well, and when he [diabetologist] said to me I wasn't, I thought, "Well that's pointless using that machine." Maybe lulling myself into a false sense of security.

Participant M1.4-They seem to place emphasis on the $\mathrm{Hb}$ [glycated haemoglobin], the three monthly reading. Erm, that was always the-an issue that I could never quite work out at the start. They would-the doctors would be more concerned about the three monthly reading, because that's what your average is, eh, and that didn't take account of my concerns, which were the big spikes I was getting. Erm, but any chat I have with the doctors nowadays, it tends to be on the, the three monthly reading and not the kind of meter readings that I have. So I guess that's maybe the evidence that they are content with for me to kind of manage and deal with the readings on a day to day basis I assume.

Participant F9.4-When he [diabetologist] said was I testing my blood and I'm saying, "Yes," y'know, "I do it twice a week, Tuesday and Saturday, before breakfast and two hours after my evening meal." And I just kind of got the feeling, y'know, nothing-no look on his face or anything - I just kind of got the feeling that was a bit much for a diabetic that's down here, y'know, when he's dealing with ones away up here, y'know. Erm, and I'm very diligent. I've kept a diary all-for all these years I kept a note of it. Participant M17.4:

Q: I'm just interested in terms of why you kind of-y'know, at what point you decided not to do that anymore. Was it something that kind of dropped off, or were you told by health professionals that it wasn't something?

A: No, no I wasn't. But I did gather the impression that they didn't really bother too much with the day to day results ... they certainly didn't, sort of, ask for these. In fact, when I did-if I told them about it they were inclined to say, "Oh it varies very much depending on whether you've had a cup of tea or a biscuit or whatever," y'know. At least, I got that impression, Liz, and it seemed to me that it-it maybe wasn't worth keeping an eye on all of this.

Participant F33.4-Why did I stop? Because it was sore and I didn't like it. And then I kept thinking, "Well I'm filling out this book, nobody ever looks at it." And you go to the doctors, and they take your blood, and they can decide from what your levels are-so why am I inflicting this pain on myself for nothing?
Demographic details of patients with type 2 diabetes $(n=20)$

\begin{tabular}{lc} 
Age (years)*: & No of patients \\
\hline $40-49$ & 5 \\
\hline $50-59$ & 1 \\
\hline $60-69$ & 11 \\
\hline$\geq 70$ years & 3 \\
\hline Sex: & 9 \\
\hline Women & 11 \\
\hline Men &
\end{tabular}

Socioeconomic statust:

\begin{tabular}{ll}
\hline I-II & 6 \\
\hline III non-manual & 5 \\
\hline III manual & 8 \\
\hline IV-V & 1 \\
\hline
\end{tabular}

Treatment at initial interview (2002-3):

\begin{tabular}{lc}
\hline Diet only & 9 \\
\hline Tablets (monotherapy) & 11 \\
\hline Tablets (combination therapy) & 0 \\
\hline Insulin and tablets & 0
\end{tabular}

Treatment at final interview (2006):

\begin{tabular}{lc}
\hline Diet only & 6 \\
\hline Tablets (monotherapy) & $5 \ddagger$ \\
\hline Tablets (combination therapy) & 8 \\
\hline Insulin and tablets & 1 \\
\hline
\end{tabular}

*Mean age at final interview 60.8 years (range 40-80).

†Using registrar general's classification system.

¥Three participants reported a dose increase.

recruitment strategy is discussed in detail elsewhere. ${ }^{26}$ We obtained ethical approval to keep participants' contact details for re-contact later if they gave written consent. Of the 21 members of the original cohort who consented, we interviewed 20 three years later in 2006 (one had died in the interim). There were no obvious differences between this subgroup and the original cohort in terms of demographic characteristics or their earlier views about self monitoring. The table provides demographic information for this subgroup.

This paper reports findings from the 18 participants who had ever self monitored their blood glucose. We coded all the data pertaining to self monitoring of blood glucose for these participants across their four interviews and examined these data looking at whether, and for what reasons, the participants' experiences of and views about self monitoring had changed over the four years. We undertook a thematic analysis, which involved repeatedly reading the extracts and then organising them into categories. Themes were compared between members of the analysis team until consensus was reached. ${ }^{27}$ Data are tagged with participants' sex, identifying number, and interview round (for example, M37.4 refers to the fourth (final) interview of male participant 37).

\section{RESULTS}

Seven of the participants were self monitoring blood glucose at the start of data collection, rising to 16 at the end of the first year. At the fourth interview, three 
years later, 10 were still regularly self monitoring. Of these, three were self monitoring twice a day two times a week (as recommended during their initial diabetes education), five were checking at least weekly or fortnightly, and one was checking three times every other day. Another participant had started taking insulin, and checked blood glucose levels at least two or three times daily. Of the eight who were not regularly self monitoring, one did so "once in a blue moon" (participant F14.4), and one did so once a month. One participant had lost his glucose meter three weeks before the interview, and the remaining five had stopped completely. Therefore, in our sample, fewer people were self monitoring at the end of the follow-up study, and, among those who still were, self monitoring was undertaken less frequently.

Three main themes emerged from our analysis: the role of health professionals; interpreting readings and managing high values; and the ongoing role of, and participants' relationship with, self monitoring.

\section{Box 2 | Interpreting readings and managing high values from blood glucose self} monitoring

Participant F15.4:

Q: And what do you think when it's higher than normal, y'know, higher than the, sort of, 6s?

A: I always says, "I should be dead" (laughs). That's what I usually say. If it's awfae [awfully] high I says, "Well I shouldnae be here." But I don't-that's what I dinnae understand either. I says, "What do you do when it's high?" She [diabetes specialist nurse] never explained that. I've asked Lyn [neighbour with type 1 diabetes] that, but she disnae ken [know] either-when you can get it up, but how do you get it down again.

Q: And nobody's really ...?

A: Nope. Nobody seems to-I don't suppose there's nothing you could do.

Participant F33.4:

Q: When you were doing them was there anything you found useful about doing it?

A: I don't think so. I mean it was just a number which really didn't make an awful lot of sense to me. And it should be between this and this, so if you were below that or you were above it, I can understand if you've got to sort of regulate all your. Like one of the girls at work; she's on insulin, so they take hers so that they can regulate her food intake and things. If it comes to that, then you have to do it because it's one of these things you have to do. But at the moment it's not. I mean it doesn't seem to have any impact on what l'm doing daily anyway.

Participant M35.3-When you monitor it, it's supposed to be between 4 and 8 . What I don't understand is when it jumps up. I know if it goes low, you eat something to bring it up. What if it goes high, what do you do to bring it down?

Participant F14.3- [In response to a high reading] I really watch what I eat. But there's a time in the day I get-I can't go for ages without something to eat. I have to have a wee snack or something.

Participant F36.4:

Q: So what are they like at the minute?

A: Fine, like 6-7, 8 even. But when it went up, it was 14-16, and I thought, "What do I do?" y'know.

Q: Yeah, yes ... were you having those readings for-consistently for a period of timethose 14-16?

A: Not con ... not every-quite often. And then I thought, "I'll need to go to the doctor and see." And that's when he put me on the metformin.

Participant M19.4-It had obviously crept up from when I started taking readings. But it got to a point where I felt, y'know, it was high both in the morning and in the evening. So that's when I took it up with the doctor.

\section{The role of health professionals}

Participants emphasised the impact that their relationships with general practitioners, diabetes nurses, and diabetologists had on their self monitoring (box 1). Participants were often provided with glucose meters in primary or secondary care settings and received initial education about how to use them. Some who had purchased their own meter reported receiving instructions from a nurse in general practice. ${ }^{19}$

None of the participants reported having been explicitly told by health professionals to stop self monitoring, nor had they received additional education about self monitoring after the first year following diagnosis. Most, however, voiced concerns about the value that health professionals placed on their readings by their fourth interview. Participants who had had contact with diabetologists had "got the feeling" (participant F9.4) "that they didn't really bother too much with the day-to-day results" (participant M17.4). Another participant commented that "there seems to be in the health service various ideas where you take it every day, you take it two times a week, or people are not bothered if you take the two month or the three month back check through your blood" (participant M37.3).

Over time, participants had gained the impression that glycated haemoglobin $\left(\mathrm{HbA}_{1 \mathrm{c}}\right)$ was the more reliable measure of glucose control on which health professionals based treatment decisions (see participant M1.4 in box 1). This led to some participants regarding self monitoring as less important as time went on, or even "pointless" (participant F8.4).

How participants viewed healthcare professionals sometimes seemed to affect their attitude to self monitoring. In our sample, older and less well educated participants articulated being particularly interested in what they perceived to be health professionals' attitudes and had, for example, stopped self monitoring because "nobody ever looks at it" (participant F33.4). Others in these groups did not engage with their readings, but simply collected data on their readings in the hope that their doctor would take an interest ("four checks in the week, I do. But I write it down, and that's as far as it goes" participant M35.4).

Self monitoring is suggested to facilitate patient empowerment, ${ }^{14}$ but it was evident from these participants' accounts that they were self monitoring for their health professionals' benefit, rather than for their own, even though the professionals did not show interest in the readings.

\section{Interpreting readings and managing high values}

Interpreting readings remained problematic for some participants (box 2), as did counterintuitive readings (such as participant F8.4 in box 3). ${ }^{19}$ Four years after diagnosis, for some, self monitoring of blood glucose "was just a number" (participant F33.4). In part, this was because no participants reported receiving ongoing education about it. Although low readings presented no problem to participants, as they knew 


\section{Box 3 Ongoing role of blood glucose self monitoring}

\section{Reasons for discontinuing}

\section{Self chastisement}

Participant F8.4:

A: I must admit if I was having, eh, an indulgence like a Chinese or something I never bother checking it (laughs) 'cos I knew it would be high.

Q: Were there any times where you checked blood sugar levels and thought, "I don't know why l'm getting this figure?"

A: Yes. Oh aye, quite a lot. Yes, but then I thought, "Well l've no medical knowledge so." But sometimes I did think, "That's a bit unfair, l've been really good," y'know.

Q: So it would be higher than you would be maybe expecting it to be?

A: Exactly. And I must admit I do find-well say, for instance, I've had my breakfast in the morning and l've had a busy day, so l've maybe not had anything to eat 'till about tea time. If I do it before it's quite low, 'cos obviously l've not eaten anything.

So I suppose it is all down to what you eat, eh.

Participant F14.2-It's telling me I'm being bad maybe or not keeping-not being strict enough—and I think, "Oh oh, I ain't using you today," or whatever. I think that's why I don't use it.

Participant F16.4:

A: Some mornings it would be great, other mornings it would be awful. Sometimes at lunch times it was (sigh); you'd think, "What have I had? Oh I had a digestive biscuit, maybe that was it." But they say you can eat a digestive biscuit, y'know. So you bla-try to think, "What on earth's caused it?" y'know.

Q: Yeah and were you about to say you blame yourself?

A: Yeah, yeah.

Q: Which is never-

A: It's not, no, y'know. You think, "Well l've not done that so it shouldnae be high," y'know. And then if you have eaten something and you are high you thought, "Well hell bloody mend you, you shouldnae have eaten that," y'know.

\section{Bodily awareness}

Participant F14.4-I'm controlling this myself. I dinnae need a machine to tell me that it's low, high, middle, what's wrong ... I ken when it's gonnae be high, and I ken when it's mediocre, and I know when it's low.

Participant M25.4-I normally, I know how I feel-whether sort of high or low, eh, and if I feel OK and take my medication, eh, I dinnae bother taking my blood.

\section{Consistency in readings}

Participant M23.4-It wasn't varying to any degree, and I stopped it really within a couple of months ... I thought, "Well what's the point?" when I'm not getting any readings that are in any way a cause for concern.

Participant M28.4-I stopped about a year ago because I was getting to the stage I was getting the same sort of levels every day ... it wasn't sort of fluctuating up and down.

\section{Reasons for continuing}

\section{Curiosity}

Participant M37.4-My average when I do my morning and evening checks is around what I need, y'know, 7s. I don't know what it is now, I'll just have a quick check for you.

Participant M1.4-I got a big slab [of cake]. I thought, "I'm going to monitor my blood after this." And I took it. Within about 40 minutes I was up to about [a] 22 blood sugar reading. Now that was proof to me that-that I needed something more than the tablets I was taking.

\section{Reassurance}

Participant F39.4-At first Dr M thought that the meter thing might be a bit fiddly for me and that, and I said, "Well I would rather have that so's I know exactly what's going on." And I do find it reassuring that when you do your check you can see right away what's registering and that.

Participant M1.4- [The meter is] there to kind of reassure me when I do sort of sense that something-I don't feel quite right. It's there to kind of give me the-reassure me it's not a hypo' coming on. That's the main thing. that eating would raise their blood glucose levels, they reported struggling to know what to do in response to high readings - ranging from puzzlement (participant M35.3) to great anxiety, as they thought high values signalled that they "should be dead" (participant F15.4).

If participants did implement a lifestyle change in response to high readings, this tended to be an instant solution rather than a permanent alteration such as long term adjustments to diet. For example, one woman commented that she temporarily stopped eating in response to high readings (participant F14.3). For some participants, the self monitoring technology seemed to promote a focus on the "here and now," which could be detrimental to long term health behaviours and decision making. Overall, participants gave little indication that they were using self monitoring to guide ongoing change to their lifestyles. Only three participants were prompted by higher readings to seek medical advice, which led to their medication being altered (such as participant M19.4). These participants were well educated men who indicated that they took an active role in consultations.

\section{The ongoing role of self monitoring}

Healthcare providers' lack of interest in their readings was the reason some gave for discontinuing self monitoring (see box 1). Moreover, the ways in which participants made either internal or external attributions for their blood glucose readings affected their attitudes to self monitoring (box 3). Ironically, most participants who had reduced or stopped self monitoring were those who made internal attributions (such as their food consumption) and blamed themselves for high readings. From a biomedical perspective, blood glucose readings are used to gain a picture of fluctuation in blood glucose levels to help understand about overall glycaemic control; however, many participants viewed and interpreted readings as a proxy measure of short term good or bad behaviour. As one participant (M25.1) said, "I must be doing all right when I get a low reading." This focus on individual behaviour often centred around diet and eating practices ${ }^{28}$ rather than other aspects of diabetes management, such as exercise or medication. Participants did not tend to attribute high readings to progression of the disease.

This association between self monitoring and individual behaviour was particularly marked for women and was closely aligned with a language of self chastisement. Women talked about avoiding self monitoring when they were eating inappropriately (such as participant F14.2 in box 3) or, conversely, taking a reading to prove their guilt about inappropriate eating (such as participant F16.4). Men, on the other hand, were more inclined to use a discourse of curiosity (such as participant M37.4), and more sanguinely attribute high readings to external causes such as their medication ("You get the odd sudden blip-like 
one day I forgot to take the tablet at the right time,' participant M19.3).

Other reasons for reducing or stopping self monitoring were participants' increased awareness of their bodily signs and symptoms (one woman used itchy skin as an indicator of high blood glucose) and having faith in their subjective knowledge of their blood glucose, and consistency in readings. Two participants reported getting stable, predictable readings and so were not gaining any new information.

Reassurance was a key reason for continuing self monitoring, and for some it seemed to have become integrated into their self image ("It's part of me now," participant F9.4) or merely routine behaviour ("Just a habit," participant F36.4).

\section{DISCUSSION}

In our sample of people with type 2 diabetes, participants attenuated self monitoring of blood glucose over time for various reasons, including health professionals' perceived disinterest in their results. Consistent, stable readings and increased awareness of physical indicators of hyperglycaemia were also reasons for discontinuing. Participants who attributed their results to external causes (such as lack of medication) were more likely to continue self monitoring than those (typically women) who blamed their own behaviour for high readings. Reassurance and habit were key reasons for continuing self monitoring. Interpreting readings and knowing how to act on high readings remained problematic, and there was little indication that participants were using self monitoring to guide or maintain behaviour change. This may help explain why studies find no association between self monitoring and glycaemic control.

Our findings suggest that the clinical uncertainty about the benefit of self monitoring of blood glucose in patients with type 2 diabetes is reflected in patients' own views about it. Our results support previous research that has indicated that patients taking oral treatment tend not to take action based on their blood glucose readings, ${ }^{29}$ but our study also suggests reasons for this. The role of health professionals is crucial, particularly as patients seem to need more guidance about interpreting and responding to readings (see box 4). ${ }^{30}$ If patients cannot understand their blood glucose fluctuations they cannot modify their behaviour. How to act on high readings was a consistent problem. Some participants had (appropriately) attenuated self monitoring because their readings were stable; ${ }^{1}$ some had stopped self monitoring because they had inferred that health professionals did not consider it important. Others continued without engaging with the results. In all cases, participants' accounts showed a lack of an explicit and unified message from their healthcare team about if, when, and how they should be self monitoring. Some practitioner guidelines advise asking for patients' interpretations of their readings, ${ }^{31}$ and this study has highlighted that patients need explicit education and

\section{Box $4 \mid$ Clinical recommendations}

- Education about self monitoring of blood glucose should be ongoing and tailored to patients' individual needs at any stage in their diabetes management

- Patients would benefit from clear, consistent signals from the different health professionals whom they consult about whether they should be self monitoring and how they should be interpreting readings

- Clear goals should be negotiated and agreed with patients

- During patient education, particular emphasis should be placed on how patients should respond to high readings, as well as low readings

- The relation between individual readings and patterns in blood glucose results should be clarified with patients

- The connections between $\mathrm{HbA}_{1 \mathrm{c}}$ and self monitoring of blood glucose should be made explicit, to enable better understanding of the relation between, and importance of, short, medium, and long term glycaemic control

dialogue about whether to continue self monitoring and how to respond to readings.

Attempts to find a concrete association between self monitoring of blood glucose and glycaemic control in patients with type 2 diabetes have investigated factors such as age, sex, duration of diabetes, and frequency of self monitoring. ${ }^{4-13}$ While our study lends some support to the relevance of sex, age and social class, it also signals that other factors may affect patients' attitudes to self monitoring. A complex interplay of both demographic and personality characteristics may influence whether patients undertake self monitoring and whether it has a positive impact on glycaemic control. We found that different individuals with similar results chose to continue or to stop self monitoring on the basis of factors such as whether they attributed readings to internal or external factors. This suggests that education should be tailored to patients' beliefs and values. Further research in homogeneous groups rather than heterogeneous populations, which takes into account the subtle interaction of personality and diabetes management style (alongside more easily measured variables) could be a fruitful avenue to pursue.

Although we studied patients' perspectives about blood glucose self monitoring, our results may apply to patient administered health technologies more generally. The strengths of this study are that it includes both newly diagnosed and veteran patients' views about self monitoring, provides an in-depth longitudinal perspective, and addresses self monitoring holistically within patients' broader views about their diabetes and health service provision. The study's weaknesses are that it relied on patients' accounts, and neither objective measures of glycaemic control nor health professionals' views were examined. We endeavoured to select a sample that reflected the spread of the population of patients with diabetes in Lothian, but, 


\section{WHAT IS ALREADY KNOWN ON THIS TOPIC}

There is no conclusive evidence for an association between self monitoring of blood glucose and improved glycaemic control in patients with type 2 diabetes

Patients' perspectives about self monitoring are largely absent from debates about the value of the practice

\section{WHAT THIS STUDY ADDS}

Patients' use of self monitoring decreases over time, informed by the perceived disinterest of health professionals towards it

Patients find results difficult to interpret and act on, and few patients use self monitoring to guide and maintain changes to their behaviour or lifestyle

Education about blood glucose self monitoring should be explicit, goal oriented, tailored to individual needs, and on a continuous basis

as with all qualitative research, our findings may not be generaliseable to other settings.

Future research could usefully explore healthcare professionals' attitudes toward self monitoring in this group, examine the information ${ }^{32}$ and guidance being provided to patients about it during consultations, and focus on the particular characteristics/personalities of patients that may make self monitoring problematic or beneficial for quality of life and glycaemic control.

We thank Margaret MacPhee for her administrative and secretarial support, the healthcare professionals who assisted with recruitment, and the people with diabetes who took part in the study. Thanks also go to the reviewers AnnLouise Kinmonth and Ulf Lindbland.

Contributors: LL, EP, and MD designed the study. EP collected the data. EP and JL analysed the data. EP drafted the paper. JL, MD, and EP were involved in the critical revisions of the paper, and approved the final version. EP is the guarantor.

Funding: This study was funded by the Chief Scientist Office, Scottish Executive Health Department. The researchers' work was independent of the funding body.

Competing interests: None declared.

Ethical approval: This study was approved by Lothian Research Ethics Committee.

1 Reynolds RM, Webb DJ. Recommendations and conclusions from a mini-symposium on self-blood glucose monitoring. J $R$ Coll Physicians Edinb 2006;36:155-8.

2 Consensus statement on self-monitoring in diabetes: Institute of Health Economics, Alberta, Canada. Int J Technol Assess Health Care 2007;23:146-51.

3 Reynolds RM, Strachan MWJ. Home blood glucose monitoring in type 2 diabetes. BMJ 2004;326:754-5.

4 Welschen LMC, Bloemendal E, Nijpels G, Dekker JM, Heine RJ, Stalman WAB, et al. Self-monitoring of blood glucose in patients with type 2 diabetes who are not using insulin: a systematic review. Diabetes Care 2005:28:1510-7.

5 Meier JL, Swislocki LM, Lopez JR, Noth RH, Bartlebaugh P, Siegel D. Reduction in self-monitoring of blood glucose in persons with type 2 diabetes results in cost savings and no change in glycemic control. Am J Managed Care 2002;8:557-65.

6 Schwedes U, Siebolds M. Meal-related structured self-monitoring of blood glucose: effects on diabetes control in non-insulin-treated type 2 diabetic patients. Diabetes Care 2002;25:1928-32.

7 Sarol JN, Nicodemus NA, Tan KM, Grava MB. Self-monitoring of blood glucose as part of a multi-component therapy among non-insulin requiring type 2 diabetes patients: a meta-analysis (1966-2004). Curr Med Res Opin 2005;21:173-83.

8 Schûtt M, Kern W, Krause U, Busch P, Dapps A, Grziwotz R, et al. Is the frequency of self-monitoring of blood glucose related to long-term metabolic control? Multicenter analysis including 24500 patients form 191 centers in Germany and Austria. Exp Clin Endocrinol Diabetes 2006;114:384-8.

9 Johnson JA, Majumdar SR, Bowker SL, Toth EL, Edwards A. Selfmonitoring in type 2 diabetes: a randomized trial of reimbursement policy. Diabet Med 2006;23:1247-51.

10 Coster S, Gulliford MC, Seed PT, Powrie JK, Swaminathan R. Selfmonitoring in type 2 diabetes mellitus: a meta-analysis. Diabet Med 2000;17:755-61.

11 Evans JMM, Newton RW, Ruta DA, MacDonald TM, Stevenson RJ, Morris AD. Frequency of blood glucose monitoring in relations to glycaemic control: observational study with diabetes database. BMJ 1999;319:83-6.

12 Abdelqadir M, Elbaqir M, Eltom M, Berne C. The influence of glucose self-monitoring on glycaemic control in patients with diabetes mellitus in Sudan. Diabetes Res Clin Pract 2006;74:90-4.

13 Franciosi M, Pellegrini F, De Berardis G, Belfiglio M, Cavaliere D, et al. The impact of blood glucose self-monitoring on metabolic control and quality of life in type 2 diabetic patients. Diabetes Care 2001;24:1870-7.

14 American Diabetes Association. Standards of medical care in diabetes: position statement. Diabetes Care 2006;29(S1):4-42.

15 Canadian Diabetes Association. Clinical practice guidelines, 2003. www.diabetes.ca/cpg2003/.

16 Karter AJ, Ackerson LM, Darbinian JA, D'Agostino RB Jr, Ferrara A, Liu J, et al. Self-monitoring of blood glucose levels and glycemic control: the Northern California Kaiser Permanente Diabetes Registry. Am J Med 2001;111:1-9.

17 National Institute of Clinical Excellence. Management of type 2 diabetes: management of blood glucose. London: NICE, 2002.

18 Farmer A, Wade A, Goyder E, Yudkin P, French D, Craven A, et al, for the Diabetes Glycaemic Education and Monitoring Trial Group. Impact of self monitoring of blood glucose in the management of patients with non-insulin treated diabetes: open parallel group randomised trial. BMJ 2007 doi: 10.1136/bmj.39247.447431.BE.

19 Peel E, Parry O, Douglas M, Lawton J. Blood glucose self-monitoring in non-insulin treated type 2 diabetes: a qualitative study of patients perspectives. BrJ Gen Pract 2004; 54:183-8.

20 Lawton J, Peel E, Douglas M, Parry O. 'Urine testing is a waste of time': newly diagnosed type 2 diabetes patients' perceptions of selfmonitoring. Diabet Med 2004;21:1045-8.

21 Parry O, Peel E, Douglas M, Lawton J. Issues of cause and control in patient accounts of type 2 diabetes. Health Educ Res 2006;21:97-107.

22 Lawton J, Parry O, Peel E, Douglas M. Diabetes service provision: a qualitative study of newly diagnosed type 2 diabetes patients preferences and views. Diabet Med 2005;22:1246-51.

23 Lawton J, Peel E, Parry O, Araoz G, Douglas M. Lay perceptions of type 2 diabetes in Scotland: bringing health services back in. Soc Sci Med 2005;60:1423-35

24 Peel E, Parry O, Douglas M, Lawton J. Diagnosis of type 2 diabetes: a qualitative analysis of patients' emotional reactions and views about information provision. Patient Educ Couns 2004;53:269-75.

25 Parry O, Peel E, Douglas M, Lawton J. Patients in waiting: a qualitative study of type 2 diabetes patients' perceptions of diagnosis. Fam Pract 2004;21:131-6.

26 Peel E, Parry O, Douglas M, Lawton J. 'It's no skin off my nose': why people take part in qualitative research. Qual Health Res 2006;16:1335-49.

27 Braun V, Clarke V. Using thematic analysis in psychology. Qual Res Psychol 2006;3:77-101.

28 Peel E, Parry O, Douglas M, Lawton J. Taking the biscuit? A discursive approach to managing diet in type 2 diabetes. J Health Psychol 2005;10:779-91.

29 Stewart D, McCaig D, Davie A, Juroszek L, Blackwood L, Findlay N, et al. Glucose self-monitoring in primary care: a survey of current practice. J Clin Pharm Ther 2004;29:273-7.

30 Skelly AH, Arcury TA, Snively BM, Bell RA, Smoth SL, Wetmore LK, et al. Self-monitoring of blood glucose in a multiethnic population of rural older adults with diabetes. Diabetes Educator 2005;31(1):8490.

31 Mayfield J, Havas S. Self-control: a physician's guide to blood glucose monitoring in the management of diabetes. Leawood, KS: American Academy of Family Physicians, 2004. (http://www.aafp.org/ 2004uspresident/PreBuilt/smbgmonograph.pdf

32 Peel E, Griffiths U, Jones S. Patients' perspectives about blood glucose self-monitoring: a qualitative evaluation of glucose monitoring advice leaflets. Diabet Med 2007;24(suppl 1):97. 